\title{
Photon Polarization Precession Spectroscopy for High-Resolution Studies of Spin Waves
}

\author{
Ralf Röhlsberger ${ }^{1,2, *}$ \\ ${ }^{1}$ Deutsches Elektronen-Synchrotron DESY, Notkestraße 85, 22607 Hamburg, Germany \\ ${ }^{2}$ The Hamburg Centre for Ultrafast Imaging, Luruper Chaussee 149, 22761 Hamburg, Germany
}

(Received 8 November 2013; published 18 March 2014)

\begin{abstract}
A new type of spectroscopy for high-resolution studies of spin waves that relies on resonant scattering of hard $\mathrm{x}$ rays is introduced. The energy transfer in the scattering process is encoded in the precession of the polarization vector of the scattered photons. Thus, the energy resolution of such a spectroscopy is independent of the bandwidth of the probing radiation. The measured quantity resembles the intermediate scattering function of the magnetic excitations in the sample. At pulsed x-ray sources, especially x-ray lasers, the proposed technique allows us to take single-shot spectra of the magnetic dynamics. The method opens new avenues to study low-energy nonequilibrium magnetic processes in a pump-probe setup.
\end{abstract}

DOI: 10.1103/PhysRevLett.112.117205

The enormous potential of fast spin manipulation for applications in information storage, processing, and retrieval stimulates a growing interest in the excited states and nonequilibrium properties of magnetic structures. The elementary quanta of excitations in an ordered ensemble of magnetic moments are magnons, also known as spin waves when described classically. Magnetic excitations are of particular interest in magnetic systems with competing interactions. For example, geometrically frustrated magnets exhibit persistent magnetic excitations even at lowest temperatures with most of their spectral weight shifted toward low energies [1,2]. This has been shown for crystalline systems [3,4] and remains an interesting subject to be studied in artificially structured systems [5,6]. Moreover, the emerging field of spin-wave engineering, also known as magnonics [7-10], relies on the understanding of low-energy magnetic excitations in nanostructured systems.

For a precise measurement of the magnetic excitation spectrum, high-resolution spectroscopic techniques are required. In the optical regime, Brillouin light scattering allows us to probe magnons with outstanding energy resolution [11-13]. The use of visible light, however, prevents the access to high momentum transfers and thus limits the range of accessible length scales. Dimensions down to interatomic distances can be reached via resonant inelastic $\mathrm{x}$-ray scattering (RIXS) or inelastic neutron scattering (INS). While single-magnon spectroscopy with $\mathrm{x}$ rays has been demonstrated just recently [14], INS for magnon studies has been an established technique for decades. In all of these methods, the energy resolution is determined (and limited) by the energy spread of the incoming particles (assuming a perfect analyzer); thus, further bandwidth reduction to achieve better energy resolution comes at the expense of the signal-to-noise ratio. Because of limited instrumental resolution, RIXS is restricted to energy transfers above $\approx 50 \mathrm{meV}$ so that the low-energy regime of magnetic excitations is still the domain of INS.
PACS numbers: 75.30.Ds, 07.85.Nc, 75.25.-j, 78.70.Ck

In fact, a very elegant decoupling of the energy resolution from the bandwidth of the probing particles has been achieved in the method of neutron spin echo (NSE) spectroscopy [15]. Small energy transfers upon inelastic scattering are encoded as phase shift in the precessing polarization of the neutrons. In combination with momentum-resolved triple-axis spectrometry [16], the dynamical structure factor of magnons can be effectively probed with $\mu \mathrm{eV}$ resolution $[17,18]$, although the energy bandwidth of the incident neutrons is much larger. As a time-of-flight method, the NSE technique relies on the finite rest mass of the neutron. Therefore, at first sight, this technique does not seem to be directly transferable to photons.

In this Letter, we introduce a new type of inelastic x-ray spectroscopy to probe magnetic excitations that exhibit similarities to NSE, the basic principle of which is illustrated in Fig. 1. The technique described here relies on resonant magnetic scattering of $x$ rays in the presence of $\mathrm{x}$-ray linear dichroism (XMLD). Under these conditions a magnetic sample with a spin wave of frequency $\Omega$ exhibits properties of a half-wave plate rotating with frequency $\Omega$. A half-wave plate reverts the angular momentum of incident circularly polarized light; thus, it constitutes the angular variant of a mirror that reverses the linear momentum of the light that is backreflected from it. In the same fashion, as photons reflected from a moving mirror experience a linear Doppler shift of $\Delta E=\mathbf{q} \cdot \mathbf{v}$, the photons transmitted through a rotating half-wave plate experience an angular Doppler shift [19-24] of $\Delta E=\mathbf{L} \cdot \Omega$, with $\mathbf{q}$ and $\mathbf{L}$ being linear and angular momentum transfer and $\mathbf{v}$ and $\Omega$ being linear and angular velocity, respectively. Note that a necessary condition for the linear and angular Doppler effect is either a broken translational invariance (interfaces, lattice planes) or a broken rotational invariance (anisotropy, optical or magnetic axes), respectively. While the linear Doppler effect forms the basis for vibrational spectroscopies, we exploit 


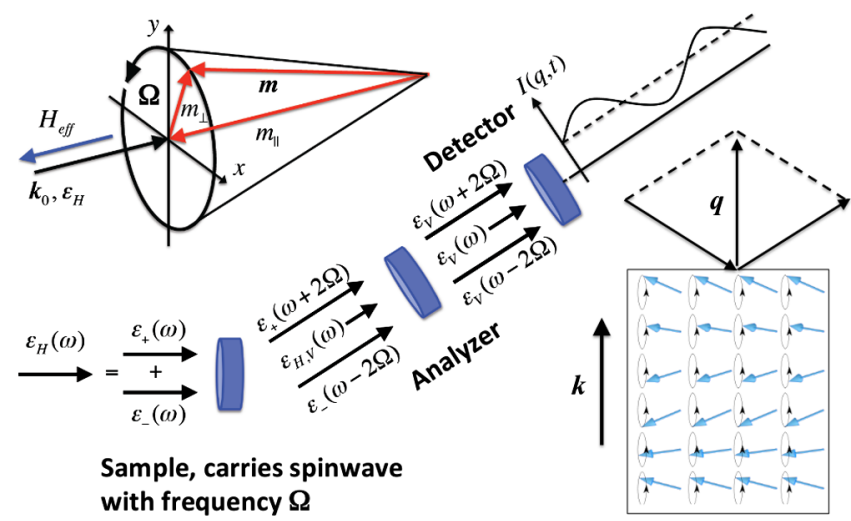

FIG. 1 (color online). Resonant scattering of linearly polarized $\mathrm{x}$ rays from a sample that carries a spin wave represented by a precessional motion with frequency $\Omega$ shown in the upper left. $\mathrm{X}$-ray magnetic linear dichroism lets the spin wave act as a rotating half-wave plate that imposes frequency shifts of $+/-2 \Omega$ on the right- or left-circular polarization components of the scattered $\mathrm{x}$ rays, respectively. After passage through a linear analyzer, a signal is observed that is modulated with frequency $2 \Omega$. In the general case of an arbitrary spin-wave spectrum, the signal is proportional to the intermediate scattering function $S(q, t)$ that probes magnetic dynamics over correlation distances $\lambda_{S}=2 \pi / q$.

here the angular Doppler effect for a new type of spectroscopy in the $\mathrm{x}$-ray regime to probe spin waves within a frequency range reaching up to $100 \mathrm{Ghz}$.

In the following, we evaluate the scattering of linearly polarized $\mathrm{x}$ rays from magnetic samples that carry spinwave excitations. We first concentrate on small momentum transfers $\mathbf{q}$ so that we can use the forward-scattering amplitude to describe the scattering process that will be treated in the kinematical approximation. The spin wave is represented by a magnetization vector $\mathbf{m}=\mathbf{m}(t)$ that precesses on a cone around the direction of the effective magnetic field, as illustrated in Fig. 1. The sample and optical elements like polarizers are described in the Jones matrix formalism by $(2 \times 2)$ matrices for a given polarization basis (e.g., linear or circular) with unit vectors $\left(\epsilon_{\mu}, \epsilon_{\nu}\right)$. We assume the photon energy being tuned to a resonance (electronic or nuclear) that is sensitive to the sample magnetization which lifts the degeneracy of the magnetic sublevels due to a spin-orbit interaction of electronic levels or a magnetic hyperfine interaction of nuclear levels.

In a resonant inelastic scattering experiment at photon energy $E=\hbar \omega_{0}$, the intensity scattered into an energy interval $d E$ and solid angle $d \Omega$ is proportional to the double-differential cross section:

$$
I_{f i} \sim \frac{d^{2} \sigma}{d E d \Omega}=\left|f\left(\mathbf{q}, \omega, \omega_{0}, \epsilon_{f}, \epsilon_{i}\right)\right|^{2} S(\mathbf{q}, \omega),
$$

where $f\left(\mathbf{q}, \omega, \omega_{0}, \epsilon_{f}, \epsilon_{i}\right)$ is the coherent atomic scattering amplitude for a given energy transfer $\hbar \omega$, momentum transfer $\mathbf{q}=\mathbf{k}_{f}-\mathbf{k}_{i}$ and change of polarization $\epsilon_{i} \rightarrow \epsilon_{f}$. $S(\mathbf{q}, \omega)$ is the dynamical structure factor of the spin wave with frequency $\omega$ and momentum q. The amplitude $f$ is derived from a $2 \times 2$ matrix that accounts for the polarization dependence of the scattering process:

$$
\mathbf{f}(\mathbf{q}, \omega)=\frac{2 \pi}{k_{0}} \sum_{i} \varrho_{i} \mathbf{M}_{i}(\mathbf{q}, \omega),
$$

where the sum runs over all atomic species in the sample. For simplicity, we drop the $\mathbf{q}$ dependence in the following and assume that the scattering proceeds close to the forward direction. $\mathbf{M}_{i}$ is then the $2 \times 2$ matrix of the coherent forward scattering length of the $i^{\text {th }}$ atomic species, and $\varrho_{i}$ is the number density of these atoms. It is convenient to decompose $\mathbf{M}(\omega)$ into a nonresonant part $\mathbf{E}(\omega)$ that describes electronic charge scattering (see the Supplemental Material [25]) and a part $\mathbf{N}(\omega)$ that contains the contributions from resonant scattering:

$$
\mathbf{M}(\omega)=\mathbf{E}(\omega)+\mathbf{N}(\omega) .
$$

With $\mathbf{m}$ denoting the unit vector of the magnetization at the position of the atom, the resonant atomic scattering length $\mathbf{N}(\omega)$ for an electric dipole transition $(L=1)$ is typically written as

$$
\begin{aligned}
{[\mathbf{N}(\omega)]_{\mu \nu}=} & \frac{3}{16 \pi}\left\{\left(\mathbf{\epsilon}_{\mu} \cdot \mathbf{\epsilon}_{\nu}\right)\left[F_{+1}+F_{-1}\right]\right. \\
& -i\left(\mathbf{\epsilon}_{\mu} \times \mathbf{\epsilon}_{\nu}\right) \cdot \mathbf{m}\left[F_{+1}-F_{-1}\right] \\
& \left.+\left(\mathbf{\epsilon}_{\mu} \cdot \mathbf{m}\right)\left(\mathbf{\epsilon}_{\nu} \cdot \mathbf{m}\right)\left[2 F_{0}-F_{+1}-F_{-1}\right]\right\},
\end{aligned}
$$

with $F_{0}, F_{+1}$, and $F_{-1}$ being the energy-dependent oscillator strengths for resonant transitions between magnetic sublevels with $\Delta m=-1,0,+1$. The three terms in Eq. (4) represent different polarization dependences. The first term is not sensitive to the sample magnetization. The second term describes circular dichroism (XMCD) because it depends on the difference between the resonant scattering amplitudes $F_{+1}$ and $F_{-1}$. Since its polarization dependence is $\mathbf{\epsilon}_{\mu} \times \mathbf{\epsilon}_{\nu}=\mathbf{k}_{0}$, it describes orthogonal scattering between the states in the polarization basis. The third term is the important one here. It describes XMLD (see Supplemental Material [25]) and is responsible for the violation of rotational invariance that gives rise to the angular Doppler effect on which the method relies.

It is convenient to express Eq. (4) in terms of $2 \times 2$ matrices within a circular polarization basis. With $C_{ \pm}=(3 / 16 \pi)\left(F_{+1} \pm F_{-1}\right) \quad$ and $\quad D=(3 / 32 \pi)\left(2 F_{0}-\right.$ $F_{+1}-F_{-1}$ ), we obtain for the scattering matrix (for derivation, see the Supplemental Material [25]): 


$$
\mathbf{M}=\left(E+C_{+}+m_{\perp}^{2} D\right) \mathbf{I}+2 m_{\|} C_{-} \mathbf{P}_{F}-m_{\perp}^{2} D \mathbf{P}_{1 / 2}(\phi)
$$

with $\mathbf{P}_{F}=\left(\begin{array}{cc}1 & 0 \\ 0 & -1\end{array}\right), \quad \mathbf{P}_{1 / 2}(\phi)=i\left(\begin{array}{cc}0 & e^{-i 2 \phi} \\ -e^{i 2 \phi} & 0\end{array}\right)$,

where $\mathbf{I}$ is the unit matrix and $\mathbf{P}_{1 / 2}(\phi)$ is the Jones matrix of a half-wave plate with the fast axis oriented at an angle $\phi$ relative to the horizontal (see Fig. 1).

In a spin wave, i.e., a collective motion of a large number of atomic magnetic moments in the sample, the magnetization $\mathbf{m}(t)$ performs precessional motion with angular frequency $\Omega$ around the effective field $\mathbf{H}_{\text {eff }}$, as illustrated in Fig. 1. In order to describe the scattering process from such an ensemble of spins, we need to perform a transformation of the scattering matrix from the corotating frame into the fixed laboratory frame. This is accomplished via the transformation

$$
\mathbf{M}(\Omega)=\mathbf{R}(\Omega) \mathbf{M}(0) \mathbf{R}^{-1}(\Omega) .
$$

$\mathbf{R}(\Omega)$ is the expectation value of the operator that generates the precessional motion of the magnetization, given by (see the Supplemental Material [25])

$$
\mathbf{R}(\Omega)=\left(\begin{array}{cc}
e^{i \Omega \cdot \mathbf{\kappa} t} & 0 \\
0 & e^{-i \Omega \cdot \mathbf{\kappa} t}
\end{array}\right) .
$$

where $\boldsymbol{\kappa}$ is a unit vector along the photon wavevector $\mathbf{k}_{0}$. Applying this transformation to the scattering matrix in Eq. (5) leaves the first two terms invariant but changes and introduces a time dependence of the third one:

$$
\mathbf{R}(\Omega) \mathbf{P}_{1 / 2}(\phi) \mathbf{R}^{-1}(\Omega)=\mathbf{P}_{1 / 2}\left(\phi_{0}+\Omega \cdot \mathbf{\kappa} t\right)
$$

i.e., the spin wave acts like a half-wave plate that rotates with angular velocity $\Omega \cdot \boldsymbol{\kappa}$. The scalar product accounts for the projection of the spin precession cone on the incident wave vector. To simplify the following discussion, we assume that $\Omega \| \mathbf{\kappa}$ so that $\Omega \cdot \mathbf{\kappa}=\Omega$ and set $\phi_{0}=0$.

In experiments with synchrotron radiation the incident field, $\mathbf{A}_{0}$ is usually linearly polarized in the horizontal plane; i.e., $\mathbf{A}_{0}=\mathbf{A}_{H}=\mathbf{\epsilon}_{H} e^{i \omega t}$. To evaluate the amplitude of the scattered field $\mathbf{A}_{S}=\mathbf{M} \mathbf{A}_{H}$, we write the incident horizontal polarization as superposition of left- and rightcircular polarization; i.e., $\mathbf{A}_{H}=\left(\mathbf{A}_{-}+i \mathbf{A}_{+}\right) / \sqrt{2}$ to obtain the following contribution from the third term in Eq. (5):

$$
\left[\mathbf{P}_{1 / 2}(\Omega t) \mathbf{A}_{H}\right]_{\text {circ }}=i e^{i 2 \Omega t} \mathbf{A}_{-}+e^{-i 2 \Omega t} \mathbf{A}_{+} .
$$

The right- or left-circular component of the incident linear polarization was converted into right- or left-circular polarization and shifted up (down) in frequency by $2 \Omega$. This means that the energy transfer $2 \Omega$ from the spin wave to the scattered photon is encoded in the relative frequency shift of the two circular components. Note that this result is independent of the carrier frequency $\omega$ so that the effective energy resolution of the method is decoupled from the frequency bandwidth of the incident radiation. Writing Eq. (9) in the linear basis yields

$$
\left[\mathbf{P}_{1 / 2}(\Omega t) \mathbf{A}_{H}\right]_{\text {lin }}=\cos 2 \Omega t \mathbf{A}_{H}-\sin 2 \Omega t \mathbf{A}_{V}
$$

which shows that the polarization performs a precessional motion in space, illustrated in Fig. 2(a). Since two orthogonal polarizations do not interfere with each other, this frequency shift is not directly observable. The interference can be induced, however, if the radiation is analyzed by a linear polarizer that projects parallel polarization components. The scattered field $\mathbf{A}_{S}$ behind a vertical analyzer with Jones matrix $\mathbf{P}_{V}$ is given by $\mathbf{A}_{S}=\mathbf{P}_{V} \mathbf{M} \mathbf{A}_{H}$. By inserting $\mathbf{M}$ given by Eq. (5) with $\phi=2 \Omega t$, we obtain (see the Supplemental Material [25])

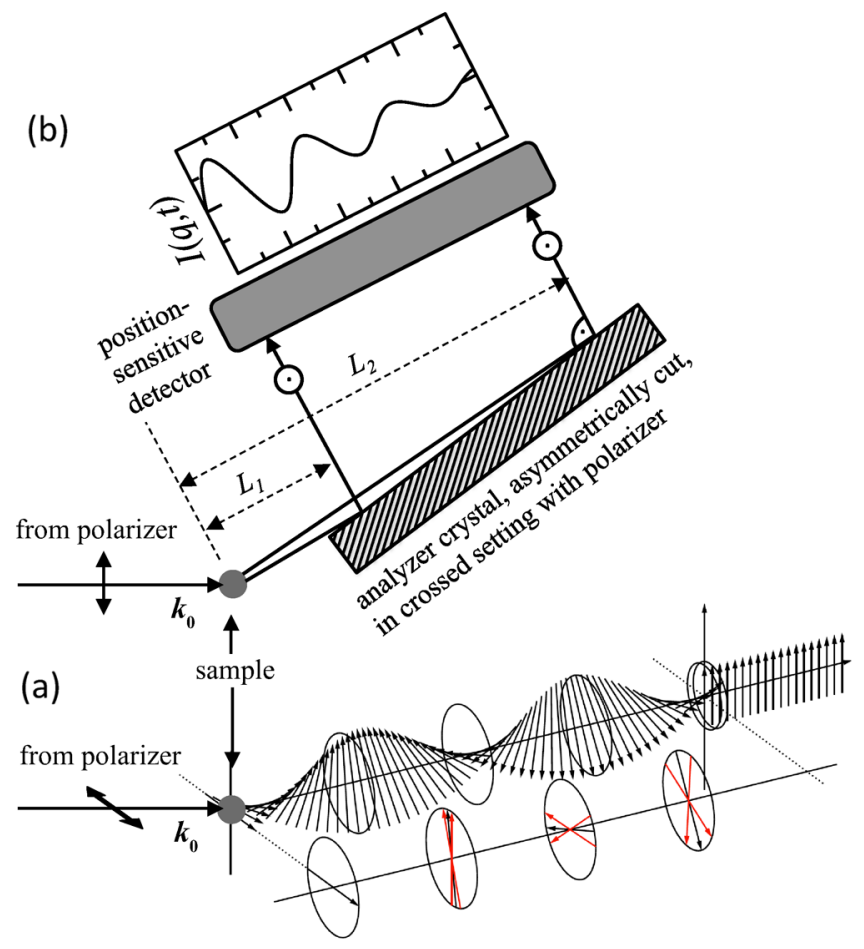

FIG. 2 (color online). (a) The photon polarization of the $\mathrm{x}$ rays scattered with momentum transfer $q$ from the spin-wave excitation (see Fig. 1) performs a precessional motion in space with a spatial period of $S=\pi c / \Omega$. The scattered photons are analyzed with a vertical polarizer that projects the vertical component of the scattered radiation, revealing the intermediate scattering function $I(q, t=L / c)$ as function of the distance $L$ from the sample. (b) Employing an asymmetrically cut analyzer crystal (Bragg angle $\Theta_{B} \approx 45^{\circ}$ ), the function can be recorded within a time interval $\left(L_{1} / c, L_{2} / c\right)$ on a position sensitive detector, potentially within a single shot of an intense pulsed x-ray source. A distribution of the spin-wave frequencies in the sample leads to a fanning out (dephasing) of the photon polarization vectors (red arrows) and thus a decreasing modulation amplitude with increasing distance from the sample. 


$$
\mathbf{A}_{S}=\left[i m_{\|} C_{-}+m_{\perp}^{2} D \sin 2 \Omega t\right] \mathbf{A}_{V}=: f(q, \Omega) \mathbf{A}_{V} .
$$

Thus, the intense, horizontally polarized direct beam that was scattered by the sample without interaction with the spin wave is completely blocked by the vertical linear polarizer. The linearly polarized wave that is transmitted by the vertical analyzer is modulated with a frequency of $2 \Omega$.

Inserting the above expression for $f(q, \Omega)$ given by Eq. (11) into Eq. (1) and integrating over all frequencies of energy loss and energy gain in the scattering process, we obtain for the intensity observed behind the analyzer:

$$
I(q, t) \approx I_{B} S(q)+I_{S} \int_{0}^{\infty}[S(q, \Omega)-S(q,-\Omega)] \sin 2 \Omega t d \Omega
$$

with $I_{B}=m_{\|}^{2}\left|C_{-}\right|^{2}$ and $I_{S}=2 m_{\|} m_{\perp}^{2} \operatorname{Re}\left[i C_{-} D^{*}\right]$, where we have used that $\left|m_{\perp}^{2} D\right|^{2} \ll\left|m_{\|} C_{-}\right|^{2}$ and that $\int S(q, \Omega) d \Omega=$ $S(q)$ being the static structure factor for given $q$. The first term in Eq. (12) is independent of $t$ and thus contributes a constant background to the measured intensity. The second term contains information about the spin-wave dynamics. It resembles the intermediate scattering function as it is obtained in NSE spectroscopy [15]. This quantity can also be obtained via time-domain interferometry based on nuclear resonant scattering [26], but the resolution and signal strength of that method are governed by the intrinsic bandwidth of the nuclear resonant scattering method.

For symmetric functions $S(q, \Omega)$ the integral in Eq. (12) vanishes. This is typically the case in the limit $\hbar \Omega \ll k_{B} T$ under the condition of detailed balance at thermal equilibrium; i.e., $S(q,-\Omega)=\exp \left(-\hbar \Omega / k_{B} T\right) S(q, \Omega)$, where the Stokes $(\Omega<0)$ and anti-Stokes $(\Omega>0)$ contributions in the spectrum are almost equal, i.e., for small energy transfers like in quasielastic scattering or at high temperatures. In magnetic systems, however, the condition of detailed balance is violated because it requires the system to possess time-reversal invariance. This is not the case in the presence of a magnetic field [27]: The equation of motion $\partial \mathbf{m} / \partial t=$ $-\gamma \mu_{0} \mathbf{m} \times \mathbf{H}_{\text {eff }}$ of the magnetization $\mathbf{m}$ in the effective field $\mathbf{H}_{\text {eff }}$ enforces only a right-handed precession. The timereverted state of a left-handed precession is not supported. Therefore, one can expect a significant asymmetry in the dynamical structure factor of magnetic systems, leading to a nonzero value for the integral in Eq. (12). This asymmetry is further enhanced with decreasing temperature. Moreover, detailed balance is significantly violated for systems that are strongly driven out of thermal equilibrium, as is the case, e.g., for spin waves that are excited by a microwave field. Since this scattering geometry with a vertical analyzer enables polarization rejection ratios up to $10^{-10}$ in a multiple reflection geometry [28-30], a very strong suppression of the nonresonant and nonorthogonal scattering can be achieved so that even weak signals can be detected with good signalto-noise ratio. This technique appears to be very attractive for studies at the $L$ edges of the REs that are constituents of materials with complex and unconventional magnetic properties and, due to their crystalline structure, should exhibit a substantial XMLD. For these energies in the range of 6-9 keV, one finds Bragg reflections of $\mathrm{Si}$ or $\mathrm{Ge}$ with Bragg angle close to $45^{\circ}$ to ensure sufficiently high polarization rejection (see the Supplemental Material [25]).

A case that is frequently encountered in experiments is a spin wave with a wave vector $\mathbf{q}$ that lies on the dispersion surface of the excitation with frequency $\Omega_{q}$ and Lorentzian line shape with half-width $\Gamma(q)$. We assume that the Stokes or anti-Stokes asymmetry for that excitation can be expressed as $S(q, \Omega)-S(q,-\Omega)=I_{0}(\Gamma / 2)^{2} /\left[\left(\Omega-\Omega_{q}\right)^{2}+\right.$ $\left.\left.(\Gamma / 2)^{2}\right]\right)$. By inserting this into Eq. (12), we obtain

$$
I(q, t)=I_{B} S(q)+I_{0} e^{-\Gamma(q) t / \hbar} \sin 2 \Omega_{q} t .
$$

The exponential results from the dephasing of the photon polarization vectors with increasing travel distance from the sample due to the distribution of spin-wave frequencies, as illustrated in Fig. 2(a). Experimentally, the time $t$ is translated into the travel distance $L$ of the photons after the scattering process, i.e., $t=L / c$, so that $I(q, t)=$ $I(q, L / c)=: I(q, L)$ can be measured via a position sensitive analyzer behind the sample, consisting of a strongly asymmetrically cut crystal with a Bragg angle equal or close to $45^{\circ}$ (the Brewster angle for hard $\mathrm{x}$ rays), as illustrated in Fig. 2(b) (see also the Supplemental Material [25]). Thus, one period $T$ of the spin wave is mapped to a distance of $L=\pi c / \Omega$. Assuming that the spatial point spread function introduced by analyzer and detector has a width of $L_{\min } \approx$ $100 \mu \mathrm{m}$ and that about 10 sampling points are required to resolve one modulation period, one finds that spin waves up to a frequency of $f_{\max }=\Omega_{\max } / 2 \pi=c /\left(20 L_{\min }\right)=$ $150 \mathrm{GHz}$ can be detected. This covers a wide range of magnetic dynamics that can be excited, e.g., via microwave fields.

It should be noted that the formalism laid out in this Letter is based on a classical description. This approach is valid either when a large number of magnons is excited in the system, e.g., via pumping by an external stimulus or when $k_{B} T \ll J S^{2}$ (with $J$ being the exchange interaction constant) but $k_{B} T \gg \hbar \Omega$. Since $\hbar \Omega \sim J S$, this can be valid only when $S \gg 1$, i.e., for large spins. This applies for many of the rare earth (RE) elements that exhibit large magnetic moments close to that of a free ion. At the $L$ edges of RE compounds and transition metal oxides, one often finds a relatively large XMLD contribution where this spectroscopy relies on. Another very interesting realization of the classical limit are collective spin-wave modes in nanoparticles in which at sufficiently low temperatures only the $q=0$ mode is populated where all spins precess in unison [31]. These modes (that can also be excited by 
microwave fields [32]) lead to peculiar magnetic properties of antiferromagnetic nanoparticles [33]. The scheme proposed here offers the possibility to record $I(q, t)$ for a given $q$ in a single shot at x-ray free-electron lasers in combination with pump-probe schemes with unprecedented energy resolution. This allows us to reveal magnetic microstates as they are populated, for example, during magnetic switching and reversal processes. If phase locked to a periodic excitation process, similar studies can be done in a stroboscopic fashion already at conventional synchrotron radiation sources. The combination with efficient microfocusing and nanofocusing of high-brilliance $\mathrm{x}$ rays allows us to uniquely access magnetic dynamics in lowdimensional systems as they are relevant in the field of spintronics and magnonics.

I acknowledge fruitful discussions with Jörg Evers, Guido Meier, Lars Bocklage, and Liudmila Dzemiantsova.

*ralf.roehlsberger@ desy.de

[1] J. S. Gardner et al., Phys. Rev. Lett. 82, 1012 (1999).

[2] G. Ehlers, J. Phys. Condens. Matter 18, R231 (2006).

[3] S. R. Dunsiger et al., Phys. Rev. Lett. 107, 207207 (2011).

[4] O. Benton, O. Sikora, and N. Shannon, Phys. Rev. B 86, 075154 (2012).

[5] J. P. Morgan, A. Stein, S. Langridge, and C. H. Marrows, Nat. Phys. 7, 75 (2011).

[6] E. Mengotti, L. J. Heyderman, A. F. Rodriguez, F. Nolting, R. V. Hügli, and H.-B. Braun, Nat. Phys. 7, 68 (2011).

[7] S. Neusser and D. Grundler, Adv. Mater. 21, 2927 (2009).

[8] V. V. Kruglyak, S. O. Demokritov, and D. Grundler, J. Phys. D 43, 264001 (2010).

[9] B. Lenk, H. Ulrichs, F. Garbs, and M. Münzenberg, Phys. Rep. 507, 107 (2011).

[10] S. O. Demokritov and A. N. Slavin, Magnonics: From Fundamentals to Applications, Topics in Applied Physics Vol. 125 (Springer, Berlin, 2013).

[11] J. R. Sanderock and W. Wettling, J. Appl. Phys. 50, 7784 (1979).

[12] B. Hillebrands, P. Baumgart, and G. Güntherodt, Appl. Phys. A 49, 589 (1989).

[13] B. Hillebrands, Light Scattering in Solids VII, edited by M. Cardona and G. Güntherodt, Topics in Applied Physics Vol. 75 (Springer, Berlin, 2000).
[14] L. Braicovich, J. van den Brink, V. Bisogni, M. M. Sala, L. J. P. Ament, N. B. Brookes, G. M. Luca, M. Saluzzo, T. Schmitt, V. N. Strocov, and G. Ghiringhelli, Phys. Rev. Lett. 104, 077002 (2010).

[15] F. Mezei, Z. Phys. 255, 146 (1972).

[16] R. Pynn, J. Phys. E 11, 1133 (1978).

[17] S. P. Bayrakci, T. Keller, K. Habicht, and B. Keimer, Science 312, 1926 (2006).

[18] B. Náfrádi, T. Keller, H. Manaka, A. Zheludev, and B. Keimer, Phys. Rev. Lett. 106, 177202 (2011).

[19] R. N. Shagam and J. C. Wyant, Appl. Opt. 17, 3034 (1978).

[20] B. A. Garetz and S. Arnold, Opt. Commun. 31, 1 (1979).

[21] B. A. Garetz, J. Opt. Soc. Am. 71, 609 (1981).

[22] V. Bagini, F. Gori, M. Santarsiero, F. Frezza, G. Schettini, and G. Schirripa Spagnolo, Eur. J. Phys. 15, 71 (1994).

[23] I. Bialynicki-Birula and Z. Bialynicka-Birula, Phys. Rev. Lett. 78, 2539 (1997).

[24] J. Courtial, D. A. Robertson, K. Dholakia, L. Allen, and M. J. Padgett, Phys. Rev. Lett. 81, 4828 (1998).

[25] See Supplemental Material at http://link.aps.org/ supplemental/10.1103/PhysRevLett.112.117205 for more details on the derivation of the scattering matrix in the presence of spin-wave excitations, the derivation of the rotational transformation matrix, and the calculation of the intensities that are measured behind horizontal and vertical analyzers.

[26] A. Q. R. Baron, H. Franz, A. Meyer, R. Rüffer, A. I. Chumakov, E. Burkel, and W. Petry, Phys. Rev. Lett. 79, 2823 (1997).

[27] A. A. Stashkevich, Y. Roussigné, P. Djemia, S. M. Chérif, P. R. Evans, A. P. Murphy, W. R. Hendren, R. Atkinson, R. J. Pollard, A. V. Zayats, G. Chaboussant, and F. Ott, Phys. Rev. B 80, 144406 (2009).

[28] T. S. Toellner, E. E. Alp, W. Sturhahn, T. M. Mooney, X. Zhang, M. Ando, Y. Yoda, and S. Kikuta, Appl. Phys. Lett. 67, 1993 (1995).

[29] E. E. Alp, W. Stuhrhahn, and T. S. Toellner, Hyperfine Interact. 125, 45 (2000).

[30] B. Marx, I. Uschmann, S. Höfer, R. Lötzsch, O. Wehrhan, E. Förster, M. Kaluza, T. Stöhlker, H. Gies, C. Detlefs, T. Roth, J. Härtwig, and G. G. Paulus, Opt. Commun. 284, 915 (2011).

[31] P. V. Hendriksen, S. Linderoth, and P. A. Lindgard, Phys. Rev. B 48, 7259 (1993).

[32] M. Grimsditch, F. Y. Fradin, Y. Ji, A. Hoffmann, R. E. Camley, V. Metlushko, and V. Novosad, Phys. Rev. Lett. 96, 047401 (2006).

[33] S. Mørup and C. Frandsen, Phys. Rev. Lett. 92, 217201 (2004) 